\title{
Prescribing errors in a Brazilian teaching hospital: causes and underlying factors from the perspective of junior doctors
}

\author{
Gislaine Bonella ${ }^{1}$, Letícia Alves ${ }^{1}$, Alicia Souza $^{2}$, and Carlos Silva ${ }^{1}$ \\ ${ }^{1}$ Federal University of Uberlandia \\ ${ }^{2}$ Federal University of Rio de Janeiro
}

June 9, 2020

\begin{abstract}
AIM In university hospital settings most prescriptions are written by junior doctors, who are more likely to make mistakes than experienced doctors. Prescribing errors can cause serious harm to patients. In Brazil, few studies have investigated the causes of these errors. Our aim was to investigate the causes of prescribing errors from the perspective of junior doctors. METHOD Qualitative, descriptive and exploratory study that used a semi-structured individual interview with questions related to the planning and execution of prescriptions. It was conducted with 34 junior doctors who graduated from twelve different universities located in six Brazilian states. The data were analyzed according to the Reason's Accident Causation model. RESULTS Among a 105 errors reported, medication omission stood out. Most errors resulted from unsafe acts during execution, followed by mistakes in planning and rule violations. Many errors reached the patients, rule violations and slips accounted for the majority. Work overload and time pressure were the most frequently reported causes. Difficulties faced by the National Health System and organizational problems of the institution were identified as latent conditions. CONCLUSION The results reaffirm the multifactorial causes of the errors. Unlike other studies, the large number of violations may be related to the misuse of junior doctors to fill gaps in the health system combined with inadequacies in the prescription and training systems. We suggest conducting other local or multicenter studies to investigate cultural and contextual aspects that help to understand the causes of prescribing errors in Brazil and other low and middle-income countries.
\end{abstract}

\section{Hosted file}

TITLE PAGE.pdf available at https://authorea.com/users/331461/articles/458154-prescribingerrors-in-a-brazilian-teaching-hospital-causes-and-underlying-factors-from-theperspective-of-junior-doctors

\section{Hosted file}

MAIN TEXT.docx available at https://authorea.com/users/331461/articles/458154-prescribingerrors-in-a-brazilian-teaching-hospital-causes-and-underlying-factors-from-theperspective-of-junior-doctors 
TITLE PAGE

\section{Conflict of Interest}

The authors have no known or potential conflicts of interest to declare. L.S.A, received a scholarship from the Scientific Initiation Scholarship Program FAPEMIG / UFU.

L.S.A and G.F.B received support for transportation and accommodation costs from the Graduate Program in Health Sciences at the Federal University of Uberlandia to participate in the "VI International Forum on Patient Safety: Medication Errors", which took place in August 2018; and in the $56^{\text {th }}$ Brazilian Congress of Medical Education (COBEM), which took place in November 2018.

\section{Ethical Approval}

The study was approved without restrictions by the Human Research Ethics Committee of the Federal University of Uberlandia on 03/28/2018, under process number 2.570.103. All participants gave their consent before participating.

\section{Financing}

There was no funding for this study.

Acknowledgments

We would like to thank all the junior doctors who offered their time to take part in this study.

\section{STATEMENTS}

What is already known about this subject:

- Drug harm differs among low, middle and high-income countries.

- Little has been studied regarding the causes of prescribing errors in low and middleincome countries. 
Box 1: Reason's accident causation model [22]

Errors, i.e., non-voluntary active failures, are divided into two classes: those deriving from failure to execute a correct plan (execution errors) and those resulting from the correct execution of an inappropriate or incorrect plan (planning error). Failures to execute a correct plan are classified into slips and lapses. The correct execution of incorrect plans, called mistakes, are classified into knowledge-based mistakes (KBMs) and rule-based mistakes (RBMs) (REASON, 2000). Violations are voluntary or intentional active failures and consist of deliberately breaking codes of conducts and routines [22]

Slips are failures to recognize and select drugs with similar names in the electronic prescription system, for example;

Lapses concern memory or attention faults, like planning the suspension of a medication and not prescribing it;

Knowledge-based mistakes (KBM) are likely to happen due to lack of knowledge or inexperience in medication prescription and refer to the incorrect choice of a plan, such as wrong medication prescription or dose due to lack of knowledge.

Rule-based mistakes (RBM), on the other hand, refer to the inappropriate choice of plan to achieve a goal. They are likely to happen due to the incorrect use of knowledge in the decision making context, for example, prescribing the dose for standard clinical conditions without considering adjustments for specific conditions such as renal function and patient age; cases where it is believed the decision at the moment is correct.

Violations are voluntary actions in which rules are deliberately ignored, for example, prescribing without a proper assessment of the patient's current clinical conditions. 
Figure 1: Active failures, error-producing and latent conditions (adapted from the Reason's Accident Causation model [22]).

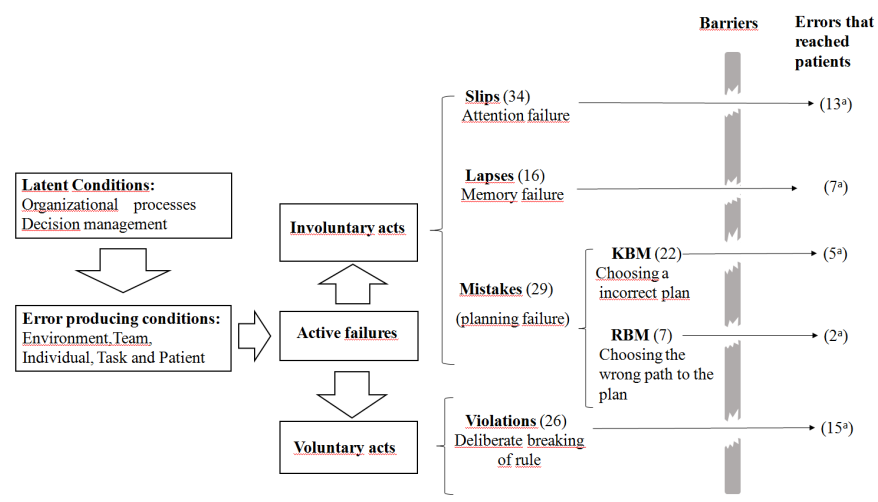

Numbers $=$ number of errors

${ }^{\mathrm{a}}=$ number of errors reaching patients

Note: the gaps in the barriers represent failures in the system

Source: the researcher 
Box 2 Examples of accounts of active faults resulting from involuntary acts. SLIP:

"The drugs had similar names, nitroglycerin and nitroprusside, vasodilators, I know the names have to be in alphabetical order, but this led me to making this mistake". Interviewee C

"Yesterday I prescribed Promethazine IM, but when I selected it for the prescription, I pressed the arrow and IV came ou without me realizing." Interviewee $Y$

Interviewee $\mathrm{S}$ forgot to suspend the thrombosis prophylaxis in the preoperative state and the surgery had to be canceled. "I've done it twice, and it makes me upset. Once we had to cancel the surgery and the other time we managed to revert the situation to allow time to operate". Interviewed $S$

"Medications that the patient takes, and you omit in the first postoperative prescription because the patient can't take it Then because you don't add the medications the first time, sometimes when you add them some are left out. This happens a lot, and sometimes you lose the continuity of some medications". Interviewee S

KBM:

Due to lack of knowledge, Interviewee $\mathrm{N}$ had to redo the prescription of magnesium sulfate for a patient with pre"When $I$.

When I started here we didn't know much about dosage, so there were times when I had to make more than one prescription for a medication due to the dilution, the saline solution, the administration, especially when it's magnesiun sulfate, because of pre-eclampsia at GO [Gynecology and Obstetrics]. We re a lithe inexpentenced when it comes to

"Lack of instructions for using some medications. We don't have a lot of knowledge on this and I don't know if it's should put it in the prescription [...] I don't know if it is something we should put in the prescription ". Interviewee U

Due to lack of knowledge, one interviewee prescribed a contraindicated drug during pregnancy.

"Once I was prescribing for a pregnant lady, and I prescribed Fluconazole, which has proven risk to pregnancy" Interviewee $R$

RBM:

Out of habit of prescribing scopolamine associated with dipyrone to all pregnant women, one of the interviewees, unconsciously and automatically prescribed it to a pregnant patient who was allergic to dipyrone.

"I was in a rush... Seeing many patients... You go to the pregnant patient.... recommend scopolamine associated with dipyrone to everyone, without knowing why and then... it was almost administrated. " $L$

"Sometimes there are two medications, IV and IM, and we end up quickly prescribing an IV medication as IM, out of habit... you rarely get an IV medication, but this is a hospital, so there are intravenous medications here where there isn " 
Table 1: Prescribing errors reported by junior doctors

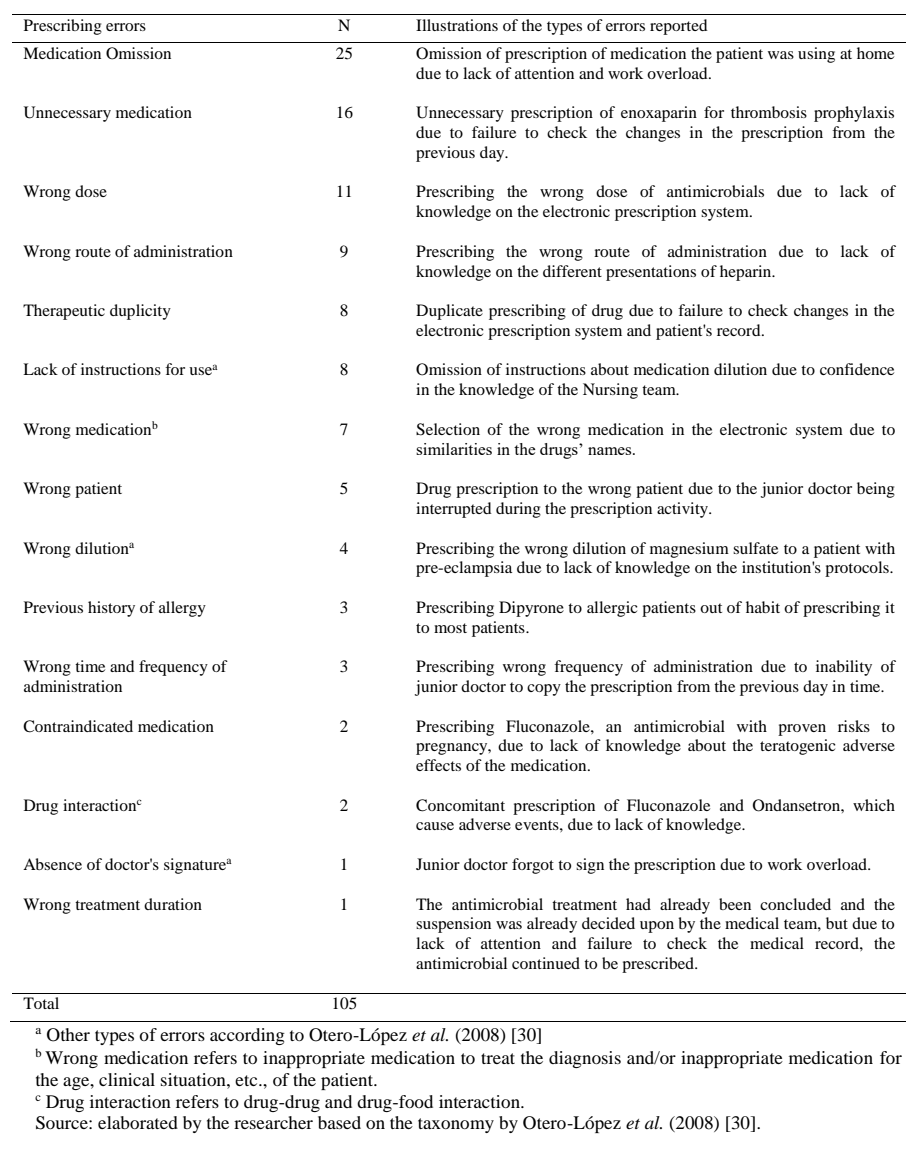


Box 3 Examples of accounts of errors resulting from voluntary acts

Violation

"... When the emergency room is full and we have to write, I don't know, thirty prescriptions until eleven in the morning... we

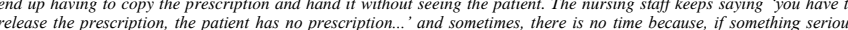
comes in the emergency room, we leave to deal with the more stable patient in the infirmary when it is possible..." (Interviewee L)

"The patient used $A A S \otimes$ and Clopidogrel $\circledast$ and did not receive them for 48 hours, because [the prescription] was copied and pasted, from Friday to Saturday and then again from Saturday to Sunday, and so there were no such medications in the

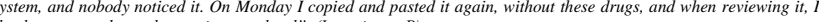
the drugs were absent the previous weekend" (Interviewee $P$ )

"Antibiotic that is suspended in a clinical round, but next day the person doesn't see it was suspended by hand the day before, and ends up just copying the prescription and prescribing it that day". Interviewee $G$

"... an erythropoietin that had been prescribed, and then the Nursing team was 'oh god, how is it administered?', I had already left, they asked the on-duty doctor, the on-duty doctor had no idea how it was used, 'let's read the drug leaflet. Then it wasn't administered. The next day they asked me: "how do you use it?", so they did not do it. One day missed because of that. (Intem 
Table 2: Classification of error-producing conditions reported by junior doctors

\begin{tabular}{lc}
\hline Error-producing conditions & N \\
\hline Environment & 21 \\
Work overload & 16 \\
Time pressure & 4 \\
Physical Environment & 2 \\
Interruptions & 1 \\
Medical Complication & \\
Team & 9 \\
Absent or inadequate tutoring & 4 \\
Communication failure & 3 \\
Trust in the Nursing team & \\
& 13 \\
\hline Individual & 5 \\
Lack of knowledge & 2 \\
Tiredness, sleepiness, hunger & \\
Inexperience & 29 \\
& \\
\hline Task & 13 \\
Electronic prescription system & 9 \\
Handwritten prescription & 3 \\
Failure to check prescriptions & 3 \\
Inadequate Protocol & \\
Unavailable or inappropriate medical records & \\
\hline Patient & \\
Complex patient or patient unfamiliar to doctor & 5 \\
Polypharmacy & 2 \\
\end{tabular}

$\mathrm{n}=$ number of times the condition was mentioned by interviewees Source: The researcher 
Box 4 Reports exemplifying error-producing conditions

"Now, what most leads you to make mistakes is, by far, the amount of work and the amount of patients". (Interviewee F)

"Being in a hurry, many patients to see, critical patients, very long prescriptions with many items, running against time to prescribe until 11 am". (Interviewee C).

"[When you are] on duty, especially in the Emergency Room, where there are lots of patients to see and patients arriving, you end up not being able to check the [patient's] history since they were admitted, and not seeing that an important
medication is missing like a prophylaxis or medication that they already used at home" (Interviewee U]

"The most common mistake I made during my training was getting the drug dilution wrong, especially when the patient is sedated or uses vasoactive drugs. Sometimes we get the dilution wrong!" How many ampoules should you
much the drip should run and for how long." This has already happened a couple of times". Interviewee $R$

"Protocols are being established now, but if a boss is from one segment and the other from another, or if the shift changes. all practices change. I'm having a lot of difficulty with this at GO [Gynecology and Obstetrics]". (Interviewee N) 
Box 5 Reports exemplifying latent conditions

'I understand that there is a reason for the copy of the prescriptions to expire, but depending on the sector where we are due to the amount of things we have to do, we are not always able to discuss the patient before prescription time and we copy it. We copy it quickly, to have time to take it for discussion, to save it, and then, after copying it every day before discussion, a lot of little things are left out." (Interviewee $K$ )

"We're used to it because the nursing team is very well trained, they know how to dilute and all. So sometimes this error happens because of that." (Interviewee H)

“... the surgical environment is stressful, the intraoperative, the preoperative. Residency in general surgery is one of the [specialties] that demand the most from junior doctors. With a [high] workload, sometimes out of choice, [because] we
know that two years is a short time, - orthopedics and other surgical specialties depend on you doing it. books don't teach you how to operate, you learn by doing -, sometimes even if you are the most dedicated [junior doctor], you miss something"(Interviewee S

"The more academic part itself. We, as a junior doctors, having more tutors, having to be less in charge of the work and have more discussions, I think that would be the way". (Interviewee F)

"Do you think we have an office to see the patient in the Emergency Room? There is no office! The patient is seen in his seat, if you are lucky to have a stretcher, they are seen on the stretcher, if not, the exam is in the seat, there is no way to
touch the abdomen, you ask embarrassing questions in front of everyone in the room . This is very serious, we have no structure to prescribe, nor to diagnose, nor anything. We know that SUS* is chaotic, that SUS has difficulties, there is no money, but [I'll tell you] one thing... this had to be shut down, because this is a crime, the patient being treated like that." money, but
(Interviewee P)

"... now when you need to look for, run after a boss to communicate a case, you have to go to the operating room... [...] and even if you say that the case is serious, this and that, depending on the boss, they won't go..." (Interviewee N)

"... recent graduates don't know how to prescribe at all, they are students, at the very beginning. It's difficult ... [...] it is not safe due to lack of tutorship, our tutorship here is very bad". (Interviewee $P$ )

"Dilutions, for example, is something we really don't learn in a systematic way. This is something that is really missing ... I know what I have [to prescribe], but how to prepare it I don 't" (Interviewee $H$ )

"I think that pharmacology, for example, is a discipline that we study a lot in the beginning [of the course] when it is a long way away from the reality of prescribing something. Then, today we need to prescribe, and no longer remember those concepts, what was taught at the time and somethings are even outdated, you know." (Interviewee V)

The discipline Pharmacology was really outdated. The basis I had as an undergraduate student was very bad for my professional life. So, it's not just my problem. Several people have the same problem." (Interviewee Z)

“... you get to work and, sometimes the boss turns around and says "copy the prescription, I'm going to stamp it", and that's not writing a prescription. I think we should have a course just to learn how to prescribe, because at least here at the
internship. it's like this: the intern doesn't write it someone dictates it to the intern without us knowing what it's for, how it works, how to prescribe it. Is this the dose used, is it just for this patient? So, if you want to learn, it's a matter of chasing them and keeping asking." (Interviewee J)

"You'll get there and see the two bosses sitting. They don't get up to look at the patient's face, and are extremely grudging when you ask for help to prescribe something." (Interviewee P)

* SUS Acronym for Sistema Único de Saúde (Unified Health System), the national health system in force in Brazil. 